\begin{tabular}{|c|c|c|}
\hline 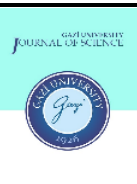 & $\begin{array}{c}\text { Gazi University } \\
\text { Journal of Science }\end{array}$ & 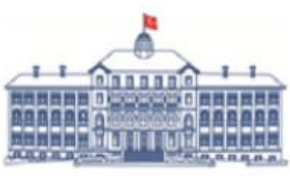 \\
\hline & http://dergipark.gov.tr/gujs & \\
\hline
\end{tabular}

\title{
Aperture Averaged Scintillation of Gaussian Beam in Strong Oceanic Turbulence
}

\author{
Muhsin Caner GOKCE* \\ TED University, Department of Electrical and Electronics Engineering, 06420, Ankara, Turkey
}

\author{
Highlights \\ - This paper focuses on aperture averaged scintillation of Gaussian beam in strong oceanic turbulence. \\ - Modified Rytov theory is employed in our study to predict scintillation. \\ - Scintillation values of modified Rytov theory are compared with that of conventional Rytov theory.
}

Article Info

Received: $10 / 05 / 2020$

Accepted: 29/08/2020

\begin{tabular}{l} 
Keywords \\
\hline Oceanic optical \\
wireless \\
communication, \\
Oceanic propagation \\
Aperture averaging \\
Scintillation
\end{tabular}

\begin{abstract}
Oceanic turbulence caused by salinity and temperature fluctuations underwater affects the characteristics of a transmit laser beam resulting in turbulence-induced intensity fluctuations (i.e., scintillation) at the receiver. Oceanic optical wireless communication (OOWC) systems employ a receiver lens of aperture to focus the collected light onto a photodetector. This way, aperture averaging takes place and the scintillation on the detector is reduced. Using the modified Rytov theory, aperture averaged scintillation of the Gaussian beam passing through strong oceanic turbulence is examined in this study. Effects of Gaussian beam parameters and the turbulence parameters on the aperture averaged scintillation and the aperture averaging factor are illustrated. The scintillation behaviors of the limiting cases of a spherical wave and a plane wave are also reported. Results show that aperture averaged scintillation decreases with increasing the size of the receiver aperture for any turbulence level. The effect of Gaussian size on the aperture averaged scintillation varies depends on the turbulence level. It is also shown that there is a close match between the point scintillation index values obtained from the modified Rytov theory and the conventional Rytov theory in low levels of turbulence.
\end{abstract}

\section{INTRODUCTION}

Oceanic optical wireless communication (OOWC) systems employ optical laser beams to transmit information-bearing signals with a huge data rate and therefore become a powerful alternative to acoustical system counterparts $[1,2]$. However, the performance of OOWC systems is deteriorated by the oceanic turbulence causing the irradiance of the information-bearing optical signal to fluctuate, thus resulting in serious errors in the received data. The fluctuations in irradiance of optical signal, also known as scintillation or fading, is quantified by the scintillation index [3]. More recently, there has been a surge of interest in exploring the scintillation index of OOWC links because the scintillation may be used as a performance criterion and gives an indication of OOWC link performance [4-10]. In this regard, scintillation indexes of Gaussian beam and its limiting forms, i.e., plane and spherical waves, are investigated with the help of the conventional Rytov theory valid under weak irradiance fluctuations [4-6]. With the extension of the conventional Rytov theory, the modified Rytov theory is introduced: in this way the scintillation indexes of the plane and the spherical waves for strong intensity fluctuations are analyzed [7]. Since bit-error-rate (BER) characterizing the quality of the communication link depends on evaluating the scintillation index, BER together with the scintillation index investigation has attracted a lot of attention for researchers of OOWC systems [11-16]. 
The turbulence-induced scintillation is mitigated by an effective technique called aperture averaging. The basic idea behind this technique is that a lens of receiver aperture is utilized at the receiver to focus the collected light onto a photodetector. This way, the fluctuations in the received intensity is averaged which provides substantial loss in the higher frequency component of the fluctuated intensity on the detector area which in turn result in reduced scintillation. In the literature, aperture averaged scintillation of the Gaussian beam and that of its limiting forms are analyzed in weak oceanic turbulence $[17,18]$. The spherical waveaperture averaged scintillation in strong oceanic turbulence is further reported in [19] where the modified Rytov theory is employed. Also, a closed-form expression of the aperture averaged scintillation for the Gaussian beam wave is derived for weak non-Kolmogorov maritime atmospheric medium and the error performance of the system is examined by the derived scintillation formula [20]. The derived formula has also offered a convenience for numerical calculations.

In this study, by considering a Gaussian beam passing through an oceanic turbulence and a finite aperture at the receiver, we evaluate the aperture averaged scintillation. Since we adopt the modified Rytov theory, findings presented in this work are valid in weak and strong turbulence levels. It should be noted that spherical wave-aperture averaged scintillation was previously reported with the help of the modified Rytov theory [19] where structure constant [21] is utilized. However in this study, the modified Rytov together with the Nikishov's turbulence spectrum model [22] is used to examine the aperture averaged scintillation of a Gaussian beam. It should also be noted that the Gaussian beam-aperture averaged scintillation in strong oceanic turbulence was utilized in the earlier studies to analyze the performance of OOWC system employing different modulation techniques [23, 24]. However, investigation of the Gaussian beam scintillation index in strong oceanic turbulence was not reported which is essential for OOWC system designers. To best our knowledge, under various oceanic turbulence scenarios, the behavior of aperture averaged scintillation of a Gaussian beam has not yet elaborately been investigated.

This study will be useful for designers of OOWC system employing Gaussian beam and operating in strong oceanic turbulence.

\section{MATERIAL METHOD}

\subsection{Power Spectrum of Oceanic Turbulence}

Under the assumption of isotropic and homogeneous oceanic water, the model for refractive index fluctuations in the turbulent ocean is defined by [22]

$$
\Phi_{n}(\kappa)=0.388 \times 10^{-8} \varepsilon^{-1 / 3} \kappa^{-11 / 3}\left(1+2.35 \kappa^{2 / 3} v^{1 / 2} \varepsilon^{-1 / 6}\right) \frac{X_{T}}{\omega^{2}}\left(\omega^{2} e^{-A_{T} \delta}+e^{-A_{S} \delta}-2 \omega e^{-A_{T S} \delta}\right) .
$$

Here, $\delta(\kappa, \eta)=8.284 \kappa^{4 / 3} v \varepsilon^{-1 / 3}+12.978 \kappa^{2} v^{3 / 2} \varepsilon^{-1 / 2}, A_{T S}=9.41 \times 10^{-3}, A_{S}=1.9 \times 10^{-4}, A_{T}=1.863 \times 10^{-2}, \omega$ is the ratio of temperature to salinity contributions to the refractive index spectrum (unitless), $X_{T}$ is the rate of dissipation of mean-squared temperature $\left(K^{2} / s\right), \varepsilon$ is the rate of dissipation of kinetic energy per unit mass of fluid $\left(\mathrm{m}^{2} / \mathrm{s}^{3}\right), v=\eta^{4 / 3} \varepsilon^{1 / 3}$ is the kinematic viscosity $\left(\mathrm{m}^{2} / \mathrm{s}\right), \eta$ being the Kolmogorov microscale length. It should be noted that the measurement values of $\omega$ can vary in the interval $[-5,0]$. The values of $\omega \rightarrow-5$ corresponds to temperature induced optical turbulence where the values of $\omega \rightarrow 0$ corresponds to salinity induced optical turbulence modelling the strong turbulence regime [25]. Furthermore, $X_{T}$ takes values from $10^{-10} \mathrm{~K}^{2} / \mathrm{s}$ in the deep ocean to $10^{-4} \mathrm{~K}^{2} / \mathrm{s}$ near water surface. From deep water to surface water (i.e., from light to dense turbulence level), $\varepsilon$ ranges from $10^{-10} \mathrm{~m}^{2} / \mathrm{s}^{3}$ to $10^{-1} \mathrm{~m}^{2} / \mathrm{s}^{3} . \eta$ is typically chosen as $\eta=1 \mathrm{~mm}$ in a region near the ocean surface. We also note that the specified values belonging to the different layers of ocean are measured by the refractometer [26]. 


\subsection{Point Scintillation in Weak Oceanic Turbulence}

In weak oceanic turbulence, the variance of irradiance fluctuations (i.e., scintillation index) is found by the conventional Rytov theory. The scintillation index of the Gaussian beam wave on the axis $\sigma_{B}^{2}$ is defined by $[27,28]$

$$
\sigma_{B}^{2}=8 \pi^{2} k^{2} L \int_{0}^{1} \int_{0}^{\infty} d \xi \kappa d \kappa \Phi_{n}(\kappa) \exp \left(-\frac{\Lambda_{1} L \kappa^{2} \xi^{2}}{k}\right)\left\{1-\cos \left[\frac{L \kappa^{2}}{k} \xi\left(1-\bar{\Theta}_{1} \xi\right)\right]\right\}
$$

where $k=2 \pi / \lambda$ is wavenumber, $\lambda$ is wavelength, $L$ is link distance, $\xi=1-z / L$ is the normalized distance parameter, $z$ is distance parameter, $\kappa$ is the spatial frequency, $\Phi_{n}(\kappa)$ is the power spectrum of refractive index fluctuations of the oceanic medium given in Equation (1), $\Lambda_{1}=\Lambda_{0} /\left(\Theta_{0}^{2}+\Lambda_{0}^{2}\right)$, $\Lambda=L /(k \alpha), \alpha_{s}$ being the Gaussian beam source size, $\Theta_{0}=1-L / F_{0}, F_{0}$ is the focal length. Here, we note that on-axis Gaussian beam scintillation index $\sigma_{B}^{2}$ reduces to plane wave scintillation index (i.e., Rytov variance) $\sigma_{R}^{2}$ when $\Theta_{1}=1$ and $\Lambda_{1}=0$ and reduces to spherical wave scintillation index when $\Theta_{1}=0$ and $\Lambda_{1}=0$.

\subsection{Aperture Averaged Scintillation in Strong Oceanic Turbulence}

The conventional Rytov theory (valid for weak fluctuations, Equation (2)), is extended to investigate the weak-to-strong fluctuations with the help of large-scale and small-scale log-irradiance variances. At the receiver, the normalized variance of power fluctuations, also known as aperture averaged scintillation index, of the Gaussian beam wave after passing through a receiver lens of circular aperture diameter $D$ is defined by the extended Rytov theory [27, 28]

$\sigma_{I}^{2}(D)=\exp \left[\sigma_{\ln X}^{2}(D)+\sigma_{\ln Y}^{2}(D)\right]-1$.

Here, $\sigma_{\ln X}^{2}(D)$ and $\sigma_{\ln Y}^{2}(D)$ denote the large-scale and small-scale log-irradiance variances and are given by Equations (4) and (5), respectively [28].

$$
\sigma_{\ln X}^{2}(D)=\frac{0.49\left(\frac{\Omega_{G}-\Lambda_{1}}{\Omega_{G}+\Lambda_{1}}\right)^{2} \sigma_{B}^{2}}{\left[1+\frac{0.4\left(2-\bar{\Theta}_{1}\right)\left(\sigma_{B} / \sigma_{R}\right)^{12 / 7}}{\left(\Omega_{G}+\Lambda_{1}\right)\left(\frac{1}{3}-\frac{1}{2} \bar{\Theta}_{1}+\frac{1}{5} \bar{\Theta}_{1}^{2}\right)^{6 / 7}}+0.56\left(1+\Theta_{1}\right) \sigma_{B}^{12 / 5}\right]^{7 / 6}},
$$

where, $\Omega_{G}=16 L / k D^{2}, \bar{\Theta}_{1}=1-\Theta_{1}, \Theta_{1}=\Theta_{0} /\left(\Theta_{0}^{2}+\Lambda_{0}^{2}\right), \sigma_{B}^{2}$ and $\sigma_{R}^{2}$ denote on-axis scintillation index of the Gaussian beam wave, and the plane wave in weak oceanic turbulence, respectively. The small-scale log irradiance variance $\sigma_{\ln Y}^{2}(D)$ in Equation (3) is defined by [28]

$$
\sigma_{\ln Y}^{2}(D)=\frac{\left(0.51 \sigma_{B}^{2}\right) /\left(1+0.69 \sigma_{B}^{12 / 5}\right)^{5 / 6}}{1+\left[1.20\left(\sigma_{R} / \sigma_{B}\right)^{12 / 5}+0.83 \sigma_{R}^{12 / 5}\right] /\left(\Omega_{G}+\Lambda_{1}\right)} .
$$




\subsection{Aperture Averaging Factor}

The aperture averaging factor (AAF) indicating how much intensity fluctuations are reduced by a circular aperture of diameter D compared with a point aperture is defined by [29]

$$
\mathrm{AAF}=\frac{\sigma_{I}^{2}(D)}{\sigma_{I}^{2}(0)} .
$$

Here, $\sigma_{I}^{2}(D)$ represents the normalized variance of signal intensity fluctuations (aperture averaged scintillation) for a finite aperture receiver with diameter $D$ and $\sigma_{I}^{2}(0)$ denotes the scintillation index on the axis for an infinitesimally small aperture $(D \approx 0)$. We note that the aperture averaged scintillation index detected by a circular aperture $\sigma_{I}^{2}(D)$ takes values less than the scintillation index detected by a infinitesimally small aperture $\sigma_{I}^{2}(0)$. Thus, AAF approaches zero when effective aperture averaging occurs.

\section{THE RESEARCH FINDINGS AND DISCUSSION}

In this section, the effect of various oceanic turbulence levels (weak and strong) on the aperture averaged scintillation index of a Gaussian beam is examined by evaluating Equation (3). We also examine the onaxis scintillation index of the Gaussian beam for comparison. In all the evaluation that follow, the wavelength of the laser source is set as $\lambda=532 \mathrm{~nm}$, focal length $F_{0}$ is fixed to infinite $F_{0}=\infty$ representing a collimated Gaussian beam, and the Kolmogorov microscale length of oceanic turbulence $\eta=1 \mathrm{~mm}$ is taken unless otherwise stated. Note that the limiting cases of the plane wave and the spherical wave presented in Figure 1 are obtained by respectively setting $\alpha_{S} \rightarrow \infty$ and $\alpha_{S} \rightarrow 0$ in Equation (3). $D=0$ represents the on-axis scintillation index of the optical wave. Also, $\sigma_{R}^{2}$ denoting the Rytov variance is used to determine the strength of turbulence. Thus, $\sigma_{R}<1$ and $\sigma_{R}>1$ correspond to weak and strong turbulence levels, respectively. The vertical dashed line drawn in Figures 1-4 represent the division between weak and strong oceanic turbulence levels. We also note that the parameter $\sigma_{R}$ is obtained by inserting $\alpha_{S} \rightarrow \infty$ into Equation (2).

In Figure 1, on-axis scintillation index versus the strength of turbulence $\sigma_{R}$ is illustrated for the plane, spherical, and the Gaussian beam waves. The area right side of the dashed line $\left(\sigma_{R}>1\right)$ is shaded to represent the strong oceanic turbulence levels. With increasing link distance (i.e., $L=[1: 1: 170]$ shown in Figure 1 mean that $L$ starts at 1 meter, ends at 170 meters with a meter increment), the scintillation index increases up to a peak value, called focusing regime, and then monotonically decreases toward a unity as the parameter $\sigma_{R}$ increases without bound. The trend observed in Figure 1 matches with the known results for strong atmospheric turbulent optical links [28]. It is interesting to note that in weak oceanic turbulence levels $\left(\sigma_{R}<1\right)$, the scintillation index values of the Gaussian beam lies between the scintillation index values of the plane and the spherical waves. However, as the turbulence level is substantially increased $\left(\sigma_{R}>5\right)$, the scintillation index of the Gaussian beam wave becomes larger than that of the plane and the spherical waves.

In Figure 2, the on-axis scintillation index predicted by the conventional Rytov theory Equation (2) (valid in weak oceanic turbulence) is compared with the on-axis scintillation index predicted by the extended Rytov theory Equation (3) (valid in weak-to-strong oceanic turbulence). Similar to Figure 1, $L$ starts at 1 meter, ends at 170 meters with three meters increments and we assume $\omega=-1, X_{T}=10^{-5} \mathrm{~K}^{2} / \mathrm{s}$, $\varepsilon=10^{-2} \mathrm{~m}^{2} / \mathrm{s}^{3}, \eta=1 \mathrm{~mm}, D=0, \lambda=532 \mathrm{~nm}, \alpha_{S}=1 \mathrm{~mm}$. It is observed that there is a close match between the on axis scintillation index values obtained from $\sigma_{B}^{2}$ and $\sigma_{I}^{2}$ for a range of $\sigma_{R}<1$ indicating that Equation (3) also gives a good prediction in weak oceanic turbulence. 


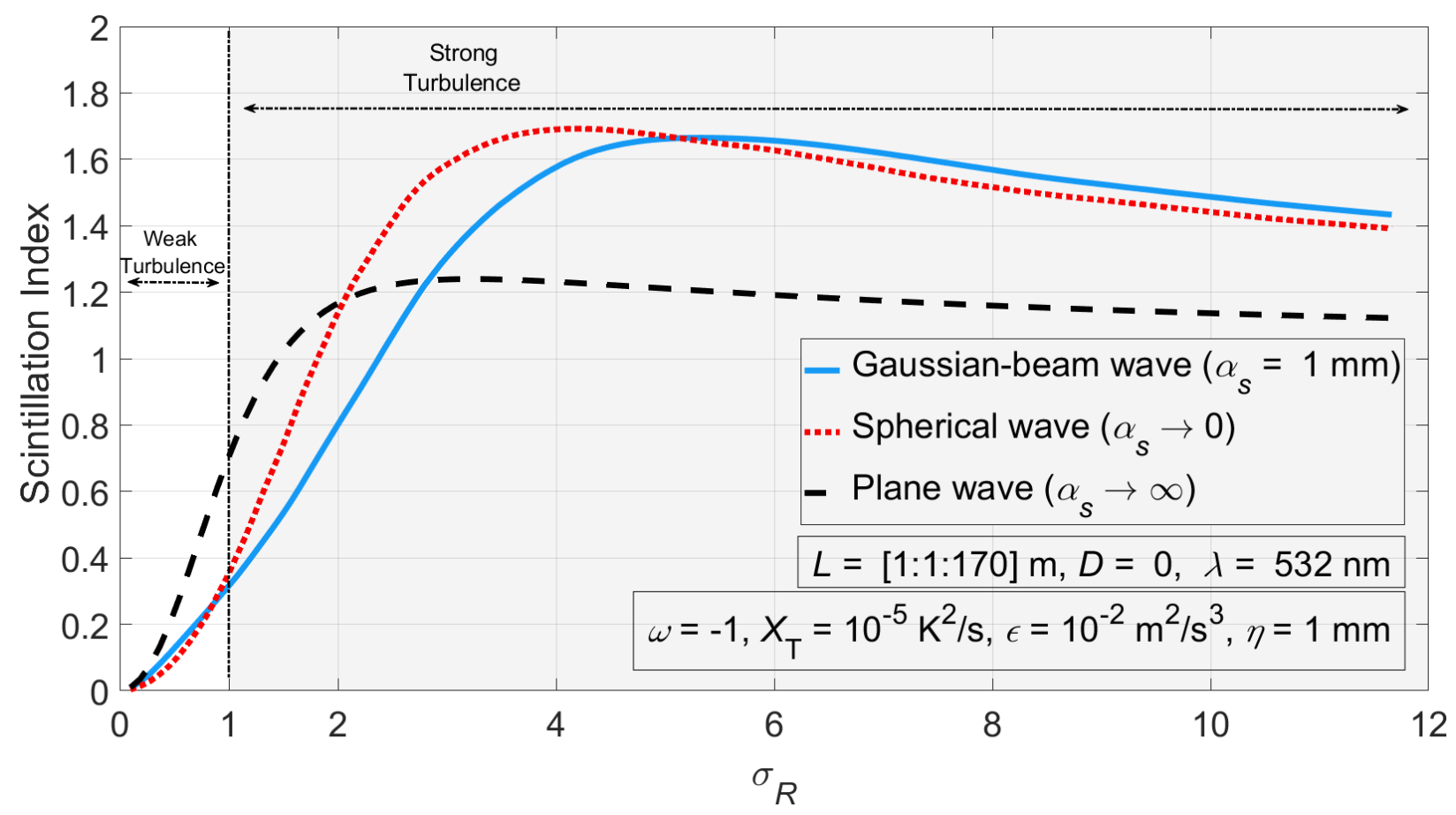

Figure 1. On-axis scintillation index of the Gaussian beam, spherical, and the plane wave $\sigma_{I}^{2}$ versus $\sigma_{R}$. The shaded area represents the strong turbulence levels

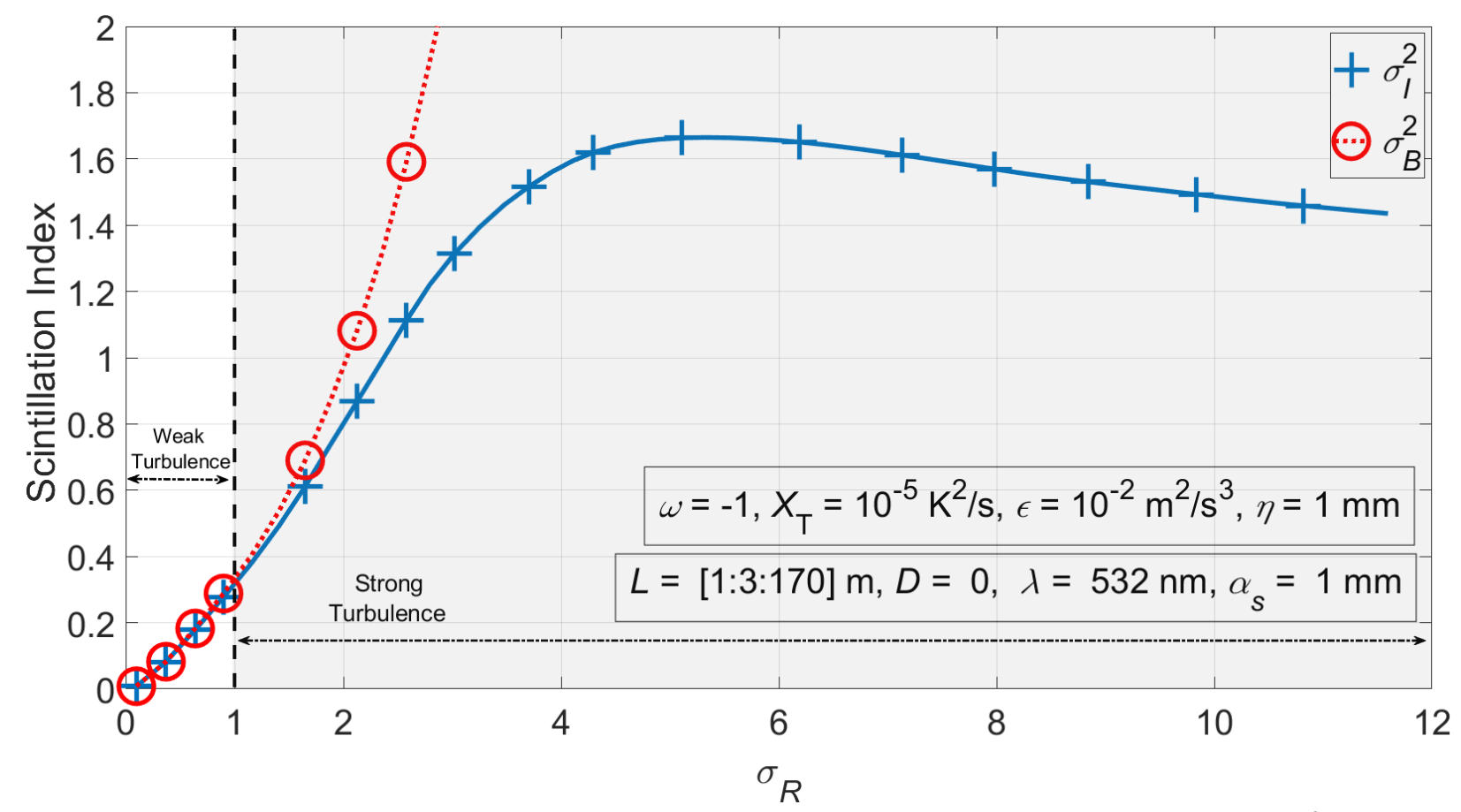

Figure 2. Scintillation index versus strength of turbulence $\sigma_{R}$ for modified Rytov theory $\sigma_{I}^{2}$ and conventional Rytov theory $\sigma_{B}^{2}$. The shaded area represents the strong turbulence levels

In Figure 3, we illustrate the effect of aperture averaging as a means of reducing the scintillation index. We note that $D=0$ corresponds to scintillation on the axis. As seen from Figure 3 , an increase in $D$ provides a substantial reduction in $\sigma_{I}^{2}(D)$ for both weak and strong oceanic turbulence levels, but the reduction is apparent in strong turbulence. The reduction in scintillation is because, fluctuations in the received intensity are averaged after passing through the collecting lens of aperture which provides substantial loss in the higher frequency component of the fluctuated intensity on the spatial plane (detector area). 
Figure 4 denotes the variation of the on-axis and the aperture averaged scintillation index versus the strength of turbulence $\sigma_{R}$ for different laser source size $\alpha_{S}$ values. The main trends of both aperture sizes are similar, within the range of $0<\sigma_{R}<2.7$, the scintillation increases with an increase in the source size. However, this behavior reverses when turbulence strength becomes $\sigma_{R}>2.7$. When the Gaussian beam propagates over a long distance where the strength of turbulence $\sigma_{R}>12$, the limiting characteristic of the plane wave is obtained. Thus, the source size variations show no effect on $\sigma_{I}^{2}(D)$.

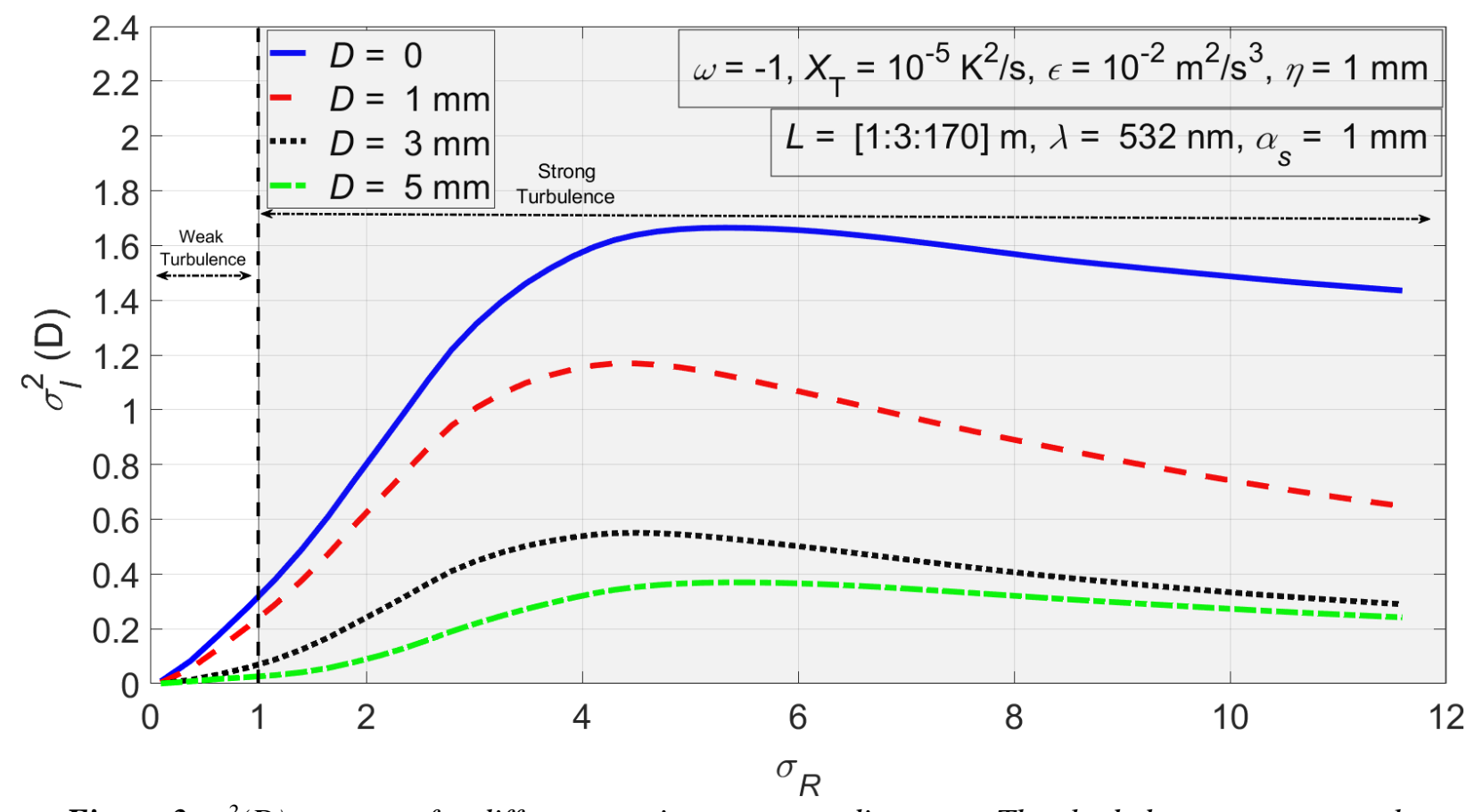

Figure 3. $\sigma_{I}^{2}(D)$ versus $\sigma_{R}$ for different receiver aperture diameters. The shaded area represents the strong turbulence levels

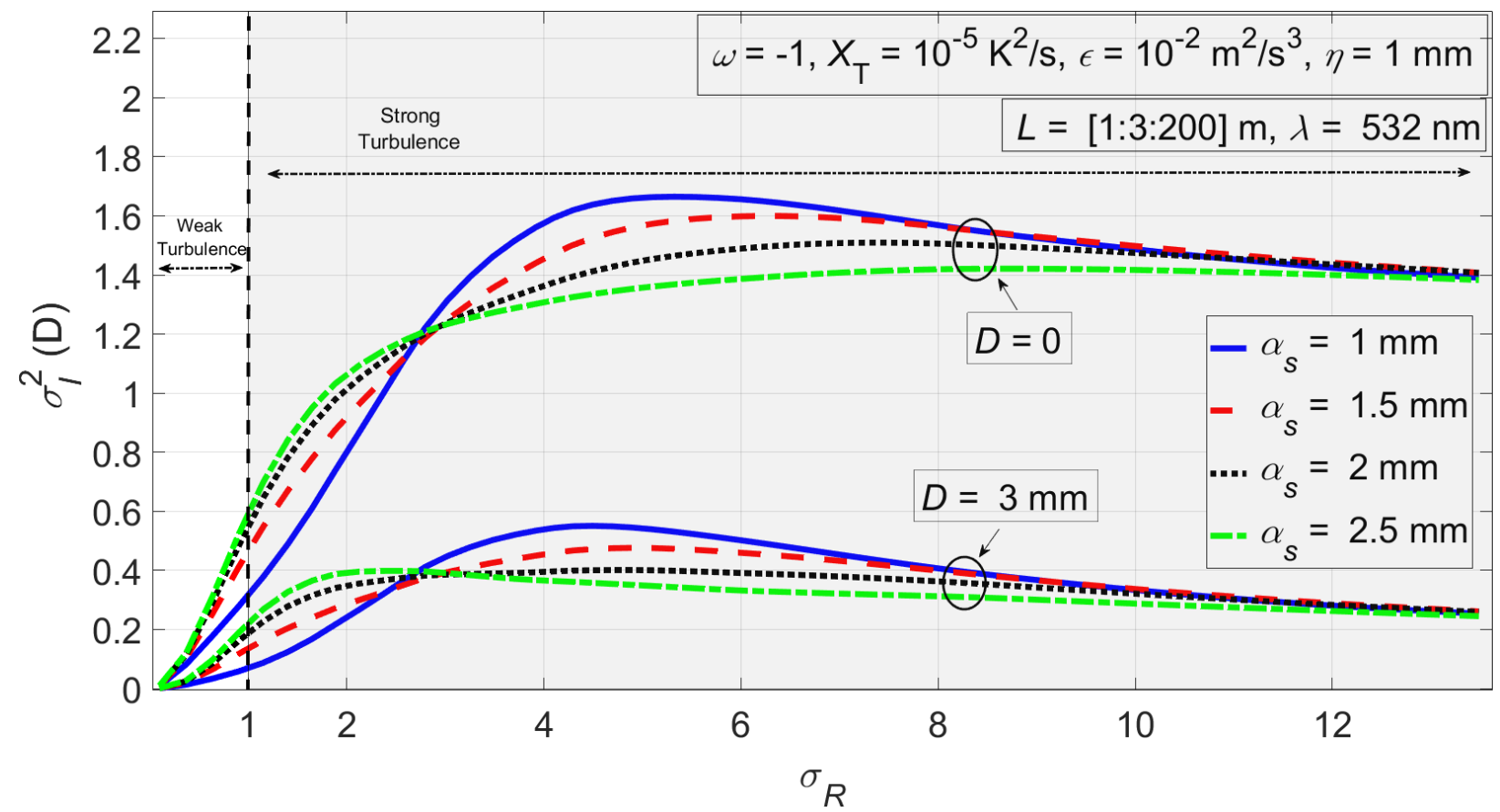

Figure 4. $\sigma_{I}^{2}(D)$ versus $\sigma_{R}$ for different source sizes $\alpha_{S}$. The shaded area represents the strong turbulence levels

Figure 5 shows the aperture averaged scintillation index versus the laser source size $\alpha_{S}$ for different aperture diameters $D$. As a benchmark, we illustrate the on-axis scintillation $(D=0)$. The main trends belonging to 
different aperture sizes are similar. At a fixed aperture diameter, with increasing source size, $\sigma_{I}^{2}(D)$ initially reduces toward the minimum level of scintillation occurring $\alpha_{S}=1.6 \mathrm{~mm}$, then increases, and eventually approach saturation for the given set of system parameters. At a fixed source size, aperture averaged scintillation decreases with increasing receiver aperture diameter. The trends observed in Figure 5 are similar to Figure 7 of [18] where we investigated the aperture averaged scintillation of the Gaussian beam in weak oceanic turbulence by conventional Rytov theory.

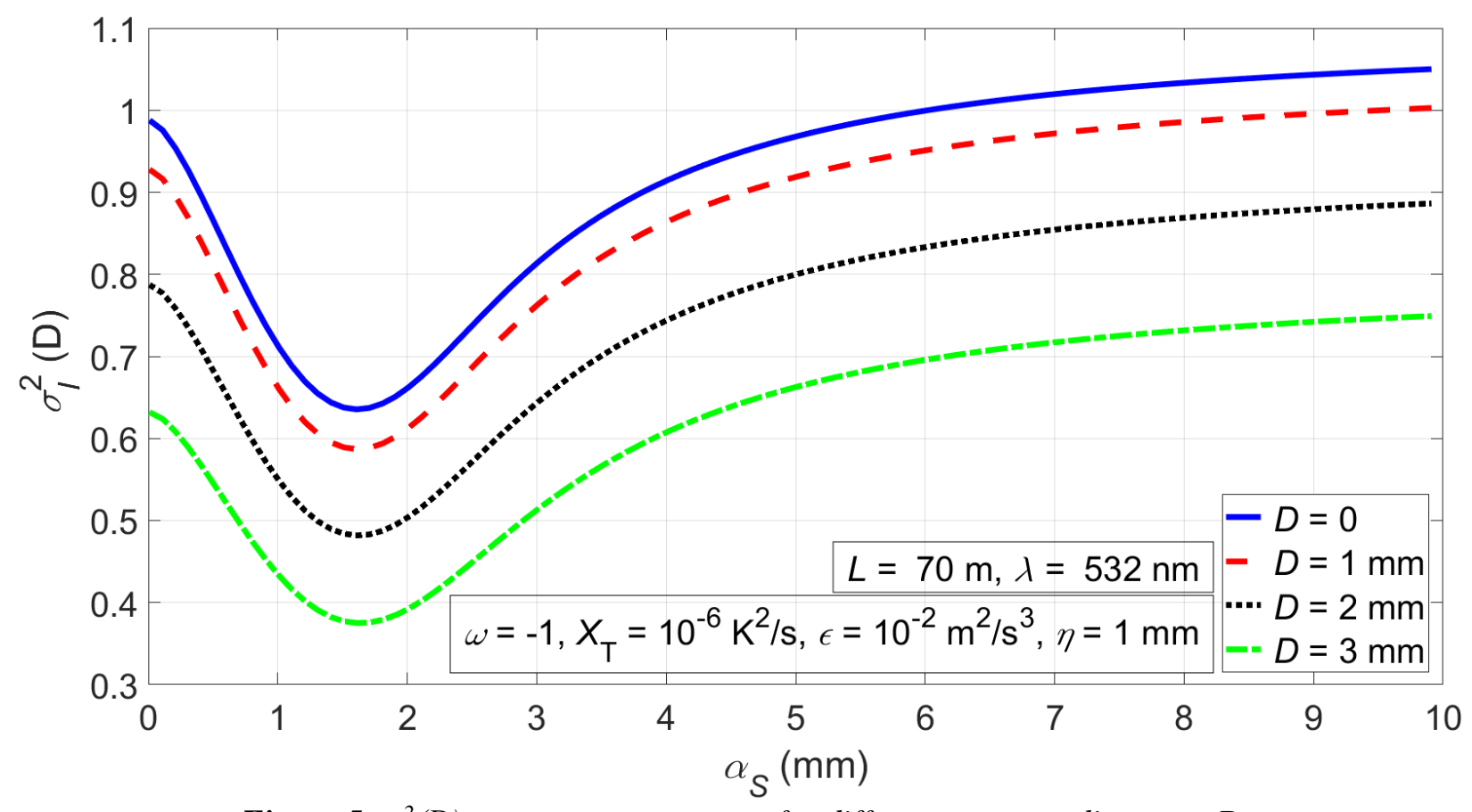

Figure 5. $\sigma_{I}^{2}(D)$ versus source sizes $\alpha_{S}$ for different aperture diameters $D$
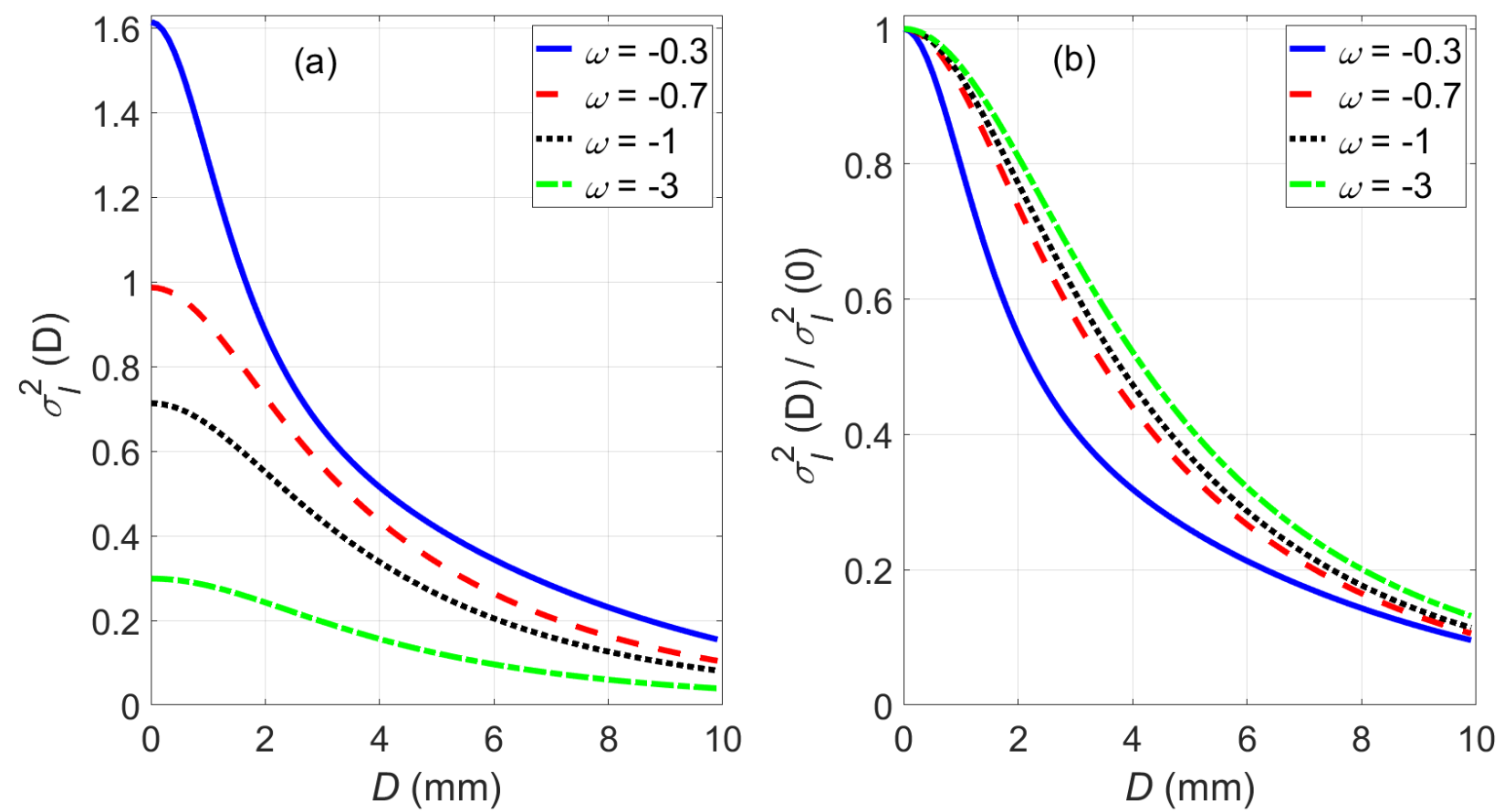

Figure 6. (a) $\sigma_{I}^{2}(D)$ versus receiver aperture diameter $D$ for different $\omega$ values. (b) AAF versus receiver aperture diameter for different $\omega$ values. The fixed parameters are $X_{T}=10^{-6} \mathrm{~K}^{2} / \mathrm{s}, \varepsilon=10^{-2} \mathrm{~m}^{2} / \mathrm{s}^{3}$, $\eta=1 \mathrm{~mm}, \alpha_{S}=1 \mathrm{~mm}, L=70 \mathrm{~m}, \lambda=532 \mathrm{~nm}$ 

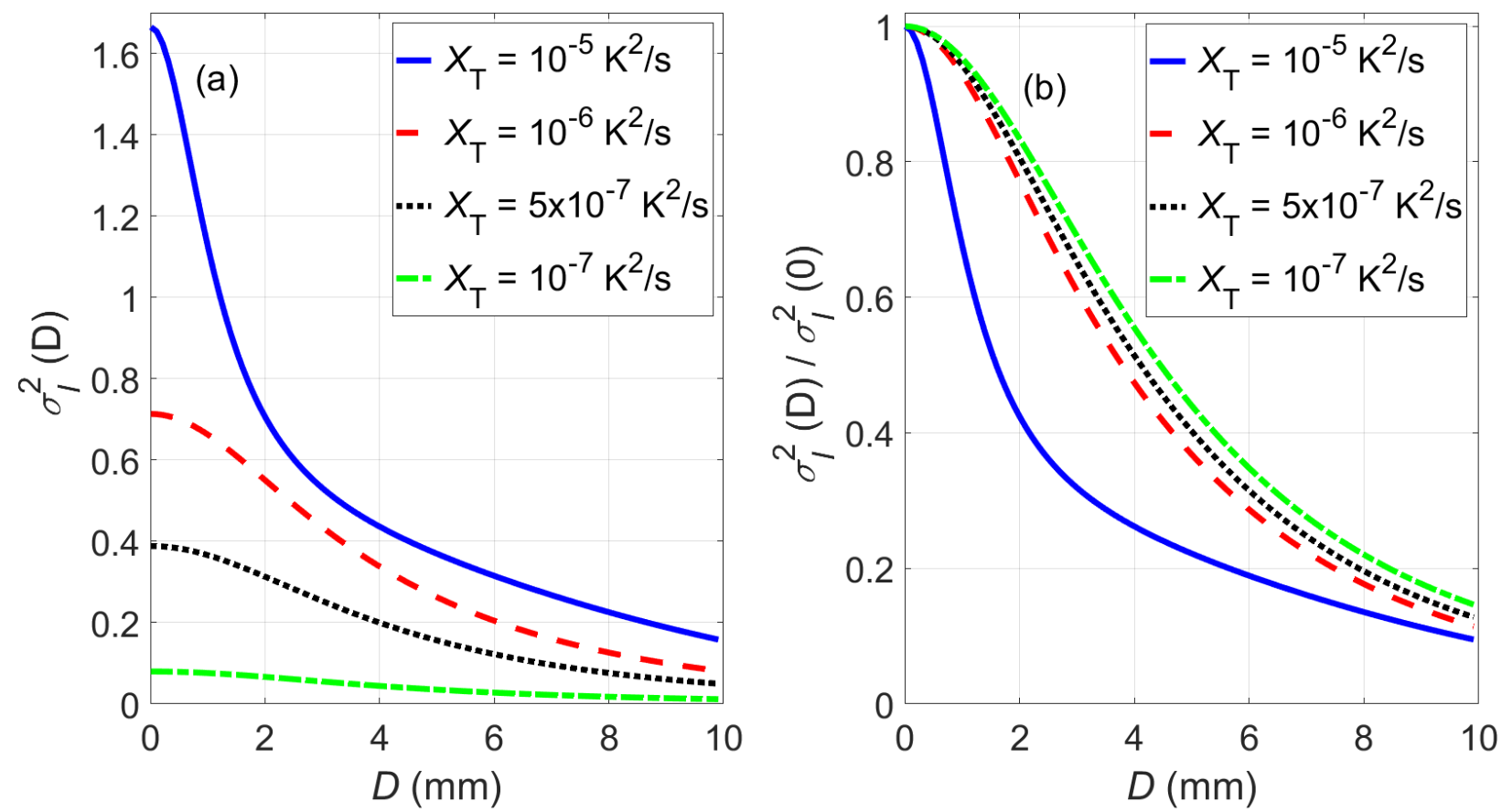

Figure 7. (a) $\sigma_{I}^{2}(D)$ versus receiver aperture diameter $D$ for different $X_{T}$ values. $(b) A A F$ versus receiver aperture diameter for different $X_{T}$ values. The fixed parameters are $\omega=-1, \varepsilon=10^{-2} \mathrm{~m}^{2} / \mathrm{s}^{3}, \eta=1 \mathrm{~mm}$, $\alpha_{S}=1 \mathrm{~mm}, L=70 \mathrm{~m}, \lambda=532 \mathrm{~nm}$
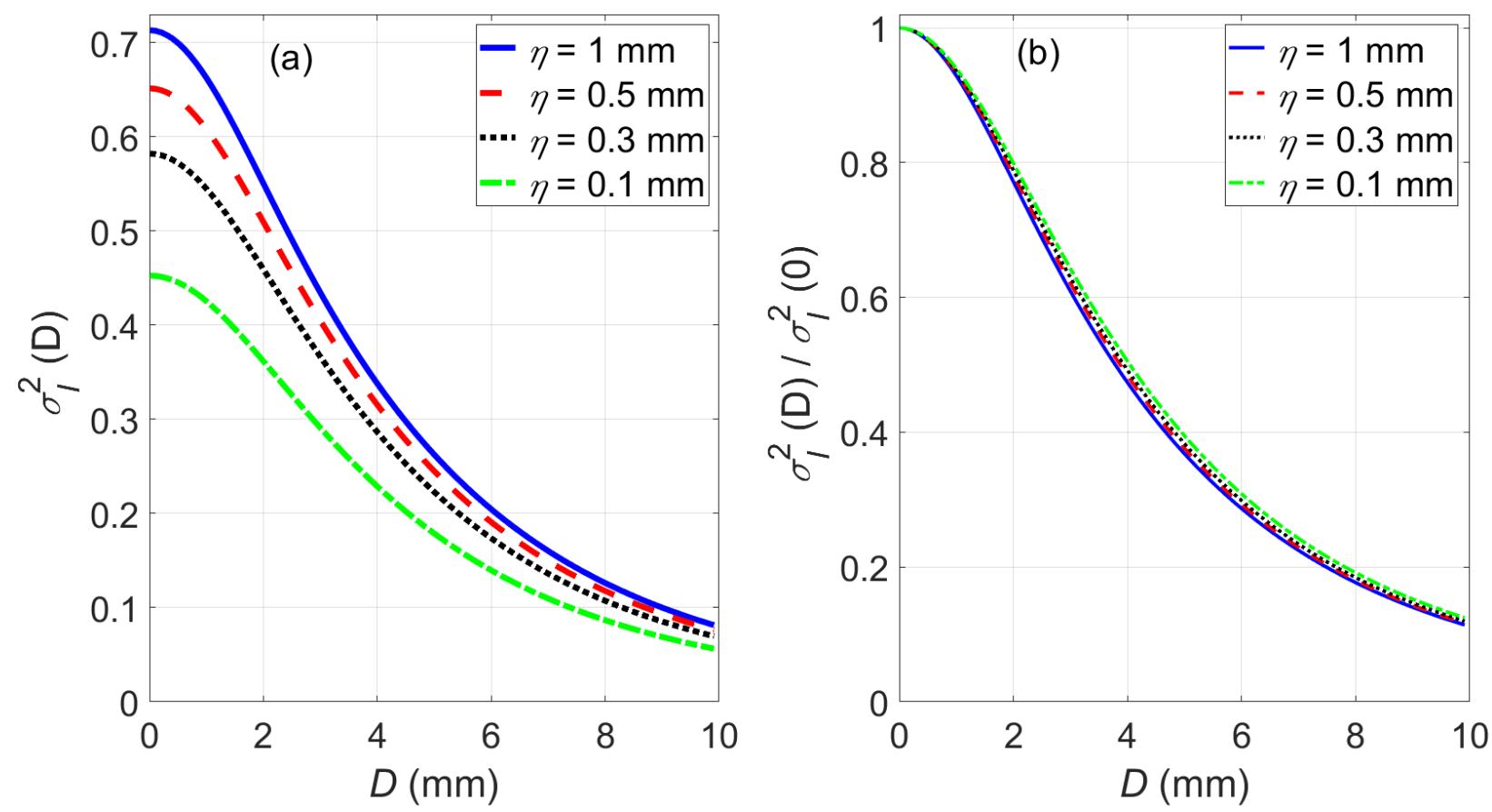

Figure 8. (a) $\sigma_{I}^{2}(D)$ versus receiver aperture diameter $D$ for different $\eta$ values. (b) AAF versus receiver aperture diameter for different $\eta$ values. The fixed parameters are $\omega=-1, X_{T}=10^{-6} \mathrm{~K}^{2} / \mathrm{s}$,

$$
\varepsilon=10^{-2} \mathrm{~m}^{2} / \mathrm{s}^{3}, \alpha_{S}=1 \mathrm{~mm}, L=70 \mathrm{~m}, \lambda=532 \mathrm{~nm}
$$

In Figures 6-9, we illustrate the variations of aperture averaged scintillation index (a) and the aperture averaging factor (b) versus receiver aperture diameter for different turbulent ocean parameters $\omega, X_{T}, \eta$, and $\varepsilon$. Note that AAF is the ratio of aperture averaged scintillation to the on-axis scintillation and helps us to understand how a receiver lens of aperture effectively average the intensity fluctuations compared to the point detector. The aperture averaged scintillation detected by a finite aperture receiver always takes values less than the on-axis scintillation detected by a point aperture and the difference between the two 
scintillation index values increases as the aperture diameter and the turbulence level increase which in turn result in a decrease in AAF values. For this reason, the small values of AAF, especially in the vicinity of zero, represent the effectively averaged (reduced)-intensity fluctuations, and this is the intended result which we want to reach. It is observed from Figures 6-9 that an increase in $\omega, X_{T}$, and $\eta$ causes $\sigma_{I}^{2}(D)$ to increase and makes AAF to decrease. However, as the rate of dissipation of kinetic energy per unit mass of fluid $\varepsilon$ increases, $\sigma_{I}^{2}(D)$ decreases, and AAF increases. The effect of Kolmogorov microscale length $\eta$ variations on $\mathrm{AAF}$ and $\sigma_{I}^{2}(D)$ is seen to be less compared to $\omega, X_{T}$, and $\varepsilon$ variations. It is understood from the figures that the effectiveness of aperture averaging starts to increase when the turbulence level increases.
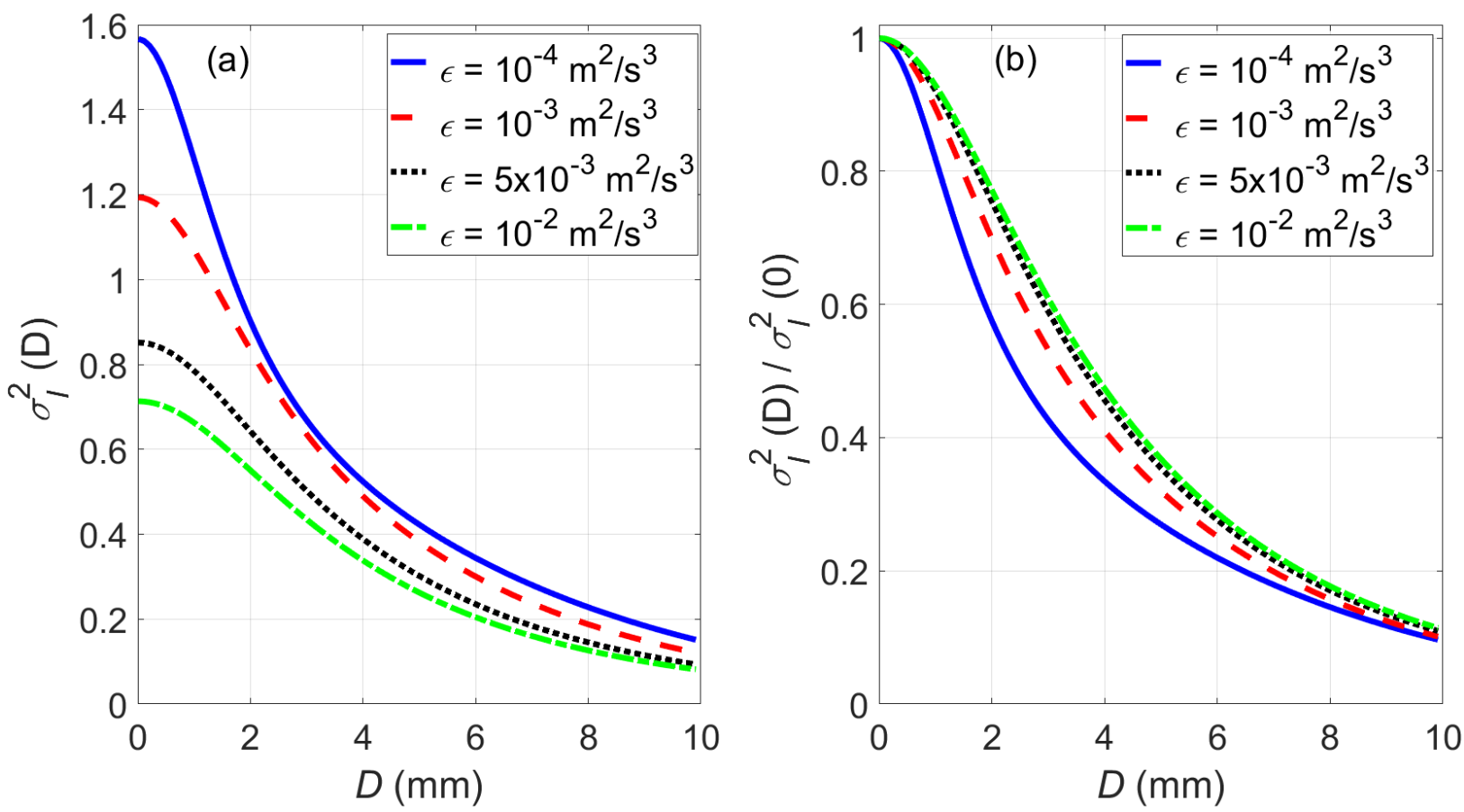

Figure 9. (a) $\sigma_{I}^{2}(D)$ versus receiver aperture diameter $D$ for different $\varepsilon$ values. (b) AAF versus receiver aperture diameter for different $\varepsilon$ values. The fixed parameters are $\omega=-1, X_{T}=10^{-6} \mathrm{~K}^{2} / s, \eta=1 \mathrm{~mm}$,

$$
\alpha_{S}=1 \mathrm{~mm}, L=70 \mathrm{~m}, \lambda=532 \mathrm{~nm}
$$

\section{RESULTS}

In weak to strong oceanic turbulence regime, we have examined the on-axis and the aperture averaged scintillation of the Gaussian beam. The scintillation index of the Gaussian beam obtained from the modified Rytov theory is compared with the scintillation index of the Gaussian beam obtained from the conventional Rytov theory. We observed that there is a close match between the on-axis scintillation index values obtained from the conventional Rytov and the modified Rytov theories under weak oceanic turbulence. This comparison shows that the validity of the conventional Rytov theory is restricted to $\sigma_{R}<1$. The findings in this paper also show that with the increase of turbulence level, the aperture averaged scintillation increases up to a peak index value in focusing regime and then monotonically decreases. A decrease in the aperture averaged scintillation and AAF is always observed when the receiver aperture diameter is increased. It is also found that a reduction in aperture averaged scintillation is observed when $\omega, X_{T}$, and $\eta$ decrease or when $\varepsilon$ increase. In strong oceanic turbulence, after a certain value of $\sigma_{R}$ is reached, aperture averaged scintillation decreases with an increase in the source size. However, aperture averaged scintillation decreases with a decrease in the source size in weak oceanic turbulence. Furthermore, at a fixed turbulence level and aperture diameter, with the increase of source size, the aperture averaged scintillation decreases toward the minimum level, then increases, and eventually saturated. AAF values obtained in strong turbulence levels are less than AAF values obtained in weak turbulence levels. Thus, we concluded that the effectiveness of aperture averaging begins to increase with increasing turbulence levels. 
Experimental works or computer simulations are required to prove that the aperture averaged scintillation index calculated by the modified Rytov theory in strong oceanic turbulence confirms measurements.

The findings in this study will help designers of the OOWC system understand the influence of strong turbulence on the Gaussian beam-aperture averaged scintillation.

\section{CONFLICTS OF INTEREST}

No conflict of interest was declared by the author.

\section{REFERENCES}

[1] Uysal, M., Capsoni, C., Ghassemlooy, Z., Boucouvalas, A., Udvary, E., "Optical Wireless Communications: An Emerging Technology", Springer International Publishing, Switzerland (2016).

[2] Zeng, Z., Fu, S., Zhang, H., Dong Y., Cheng, J., "A Survey of Underwater Optical Wireless Communications", IEEE Communications Surveys \& Tutorials, 19(1): 204-238, (2017).

[3] Korotkova, O., Random Light Beams: Theory and Applications, CRC Press, Boca Raton, FL, (2014).

[4] Ata, Y., Baykal, Y., "Scintillation of optical plane and spherical waves in underwater turbulence", J. Opt. Soc. Am. A, 31(7): 1552-1556, (2014).

[5] Korotkova, O., Farwell, N., Shchepakina, E., "Light scintillation in oceanic turbulence", Waves in Random and Complex, 22(2): 260-266, (2012).

[6] Wang, Z., Zhang, P., Qiao, C., Lu, L., Fan, C., Ji X., "Scintillation index of Gaussian waves in weak turbulent ocean", Opt. Commun., 380: 79-86, (2016).

[7] Baykal, Y., "Scintillation index in strong oceanic turbulence”, Opt. Commun., 375: 15-18, (2016).

[8] Baykal, Y., "Intensity fluctuations of multimode laser beams in underwater medium”, J. Opt. Soc. Am. A, vol. 32(4): 593-598, (2015).

[9] Baykal, Y., "Higher order mode laser beam scintillations in oceanic medium", Waves in Random and Complex Media, 26(1): 27-29, (2016).

[10] Baykal, Y., "Scintillation of LED sources in underwater medium", Appl. Opt., 55(31): 8860-8863, (2016).

[11] Gerçekcioğlu, H., "Bit error rate of focused Gaussian beams in weak oceanic turbulence", J. Opt. Soc. Am. A, 31(9): 1963-1968, (2014).

[12] Arpali, S. A., Baykal, Y., Arpali, Ç., "BER evaluations for multimode beams in underwater turbulence", J. Mod. Opt., 63(13): 1297-1300, (2016).

[13] Gökçe, M. C., Baykal, Y., "Scintillation analysis of multiple-input single-output underwater optical links", Appl. Opt. 55(22): 6130-6136, (2016).

[14] Baykal, Y., "Bit error rate of pulse position modulated optical wireless communication links in oceanic turbulence", J. Opt. Soc. Am. A, 35(9): 1627-1632, (2018). 
[15] Wu, T., Ji, X., Zhang, H. Li, X., Wang, L., Fan, X., "Rytov variance of spherical wave and performance indicators of laser radar systems in oceanic turbulence", Optics. Commun., 434: 3643, (2019).

[16] Sari F., Yenice, Y. E., "Lorentz-Gaussian beam performance for weak turbulence", International Conference on Broadband Communications for Next Generation Networks and Multimedia Applications (CoBCom), Graz, 1-4, (2016).

[17] Yi, X., Li, Z., Liu, Z., "Underwater optical communication performance for laser beam propagation through weak oceanic turbulence", Appl. Opt., 54(6): 1273-1278, (2015).

[18] Gökçe, M. C., Baykal, Y., "Aperture averaging and BER for Gaussian beam in underwater oceanic turbulence", Opt. Commun., 410(5): 830-835, (2018).

[19] Gökçe, M. C., Baykal, Y., “Aperture averaging in strong oceanic turbulence”, Opt. Commun., 413(7): 196-199, (2018).

[20] Cheng, M., Guo, L., Zhang Y., "Scintillation and aperture averaging for Gaussian beams through non-Kolmogorov maritime atmospheric turbulence channels," Opt. Express, 23(25): 3260632621, (2015).

[21] Baykal, Y., "Expressing oceanic turbulence parameters by atmospheric turbulence structure constant", Appl. Opt., 55(6): 1228-1231, (2016).

[22] Nikishov, V. V., Nikishov, V. I., "Spectrum of turbulent fluctuations of the sea-water refraction index”, Int. J. Fluid Mech. Res., 27(1): 82-98, (2000).

[23] Gökçe, M. C., Baykal, Y., Ata, Y., "Performance analysis of M-ary pulse position modulation in strong oceanic turbulence", Opt. Commun., 427: 573-577, (2018).

[24] Gökçe, M. C., Baykal, Y., Ata, Y., "M-ary phase shift keying-subcarrier intensity modulation performance in strong oceanic turbulence", Opt. Eng., 58(5): 056105, (2019).

[25] Wang, Z. Lu, L., Zhang, P., Qiao, C., Zhang, J., Fan, C., Ji, X., "Laser Beam Propagation through Oceanic Turbulence", IntechOpen (2018).

[26] Alford, M. H., Gerdt D. W., Adkins C. M., "An ocean refractometer: resolving millimeter-scale turbulent density fluctuations via the refractive index", J. Atmos. and Ocean. Tech., 23(1): 121137, (2006).

[27] Andrews, L. C., Phillips, R. L., Hopen, C. Y., "Laser Beam Scintillation with Applications", SPIE Press, Bellingham, Washington (2001).

[28] Andrews, L. C., Phillips, R. L., "Laser Beam Propagation through Random Media", SPIE, Bellingham, Washington (2005).

[29] Wang, S. J., Baykal, Y., Plonus, M. A., "Receiver aperture averaging effects for the intensity fluctuation of a beam wave in the turbulent atmosphere", J. Opt. Soc. Am., 73(6): 831-837, (1983). 